\title{
Scaling Data Analytics with Moore's Law
}

\author{
Kunle Olukotun \\ Pervasive Parallelism Laboratory \\ Stanford University
}

\begin{abstract}
Analyzing the volume, variety and velocity of big data requires the use of modern heterogeneous computing platforms composed of multicores with SIMD execution units, GPUs, clusters, FPGAs and in the future new reconfigurable architectures. However, programming in this environment is extremely challenging due to the need to use multiple low-level programming models and then combine them together in ad-hoc ways. Furthermore, many data analytics algorithms do not take full advantage of modern hardware capabilities. To optimize big data applications both for modern hardware and for modern programmers needs algorithms specialized for modern hardware and a high-level programming model that executes efficiently on heterogeneous parallel hardware. In this talk, I will describe the Delite DSL framework, which uses nested parallel patterns encapsulated in domain specific languages (DSLs). I will describe how a nested parallel pattern based programming model can be used to develop new data analytics algorithms that are optimized for architectures as diverse as multicore/NUMA, clusters, GPUs, FPGAs and a new reconfigurable architecture called Plasticine.
\end{abstract}

Author Keywords: Data Analytics, Heterogeneous architectures, Domain Specific Programming Languages, Reconfigurable Architectures.

\section{Short Bio}

Kunle Olukotun is the Cadence Design Systems Professor in the School of Engineering and Professor of Electrical Engineering and Computer Science at Stanford University.

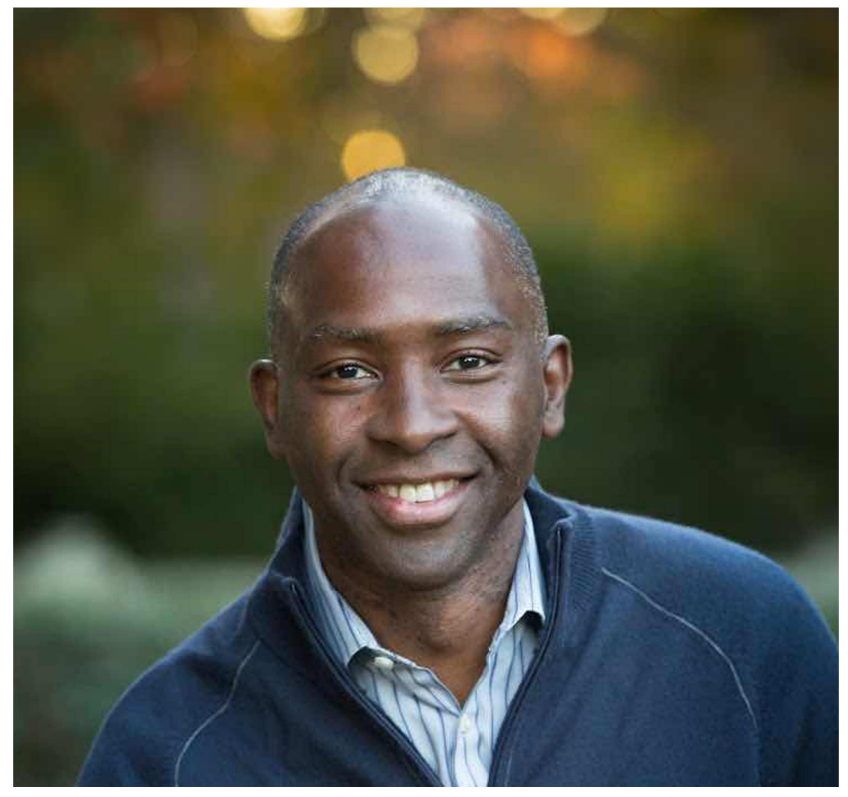

Olukotun is well known as a pioneer in multicore processor design and the leader of the Stanford Hydra chip mutlipocessor (CMP) research project. Olukotun founded Afara Websystems to develop high-throughput, low-power multicore processors for server systems. The Afara multicore processor, called Niagara, was acquired by Sun Microsystems. Niagara derived processors now power all Oracle SPARC-based servers. Olukotun currently directs the Stanford Pervasive Parallelism Lab (PPL), which seeks to proliferate the use of heterogeneous parallelism in all application areas using Domain Specific Languages (DSLs). Olukotun is an ACM Fellow and IEEE Fellow.
Permission to make digital or hard copies of part or all of this work for personal or classroom use is granted without fee provided that copies are not made or distributed for profit or commercial advantage and that copies bear this notice and the full citation on the first page. Copyrights for thirdparty components of this work must be honored. For all other uses, contact the Owner/Author. Copyright is held by the owner/author(s).

PACT'16, September 11-15, 2016, Haifa, Israel.

ACM ISBN 978-1-4503-4121-9/16/09.

DOI: http://dx.doi.org/10.1145/2967938.2970375 